\title{
INICIACIÓN SEXUAL Y CONSTRUCCIÓN DEL DESEO EN VARONES CON PRÁCTICAS HOMOERÓTICAS EN EL EJE CAFETERO COLOMBIANO
}

\author{
Gabriel Gallego Montes \\ Universidad de Caldas \\ Sebastián Giraldo Aguirre \\ Universidad de Caldas
}

\section{RESUMEN}

Este artículo presenta un acercamiento a la iniciación sexual entre varones con prácticas homoeróticas en el Eje Cafetero colombiano. La revisión de literatura evidencia el predominio de una mirada epidemiológica asociada al VIH/SIDA en los estudios sobre sexualidad juvenil. La investigación fue llevada a cabo en el año 2012, a través de una encuesta biográfica a 401 hombres, en las ciudades de Manizales, Pereira, Armenia y Cartago. Los hallazgos detallan la construcción del deseo y la primera experiencia sexual, sus contextos de ocurrencia y una relación entre prácticas sexuales y diferencias de edad con la pareja. Como conclusión puede advertirse que la construcción del deseo y las prácticas sexuales conjugan de manera problemática los campos del homoerotismo y heterosexualidad.

\section{PALABRAS CLAVE}

Iniciación sexual, Homoerotismo, Varones, Homosexualidad masculina, Biografías sexuales, Colombia

\section{ABSTRACT}

This paper discusses an approach to sexual initiation among men with homoerotic practices in the Colombian Coffee Landscape. A literature review evidence the predominance of an epidemiological approach in studies on youth sexuality associated with HIV/AIDS. The study was conducted in 2012 by a biographical survey with 401 men, in the cities of Manizales, Pereira, Armenia and Cartago. The findings detail the construction of desire and the first sexual experience, their contexts of occurrence 
and a relationship between sex and age differences with the couple. In conclusion, the construction of desire and sexual practices combine problematically homoeroticism fields and heterosexuality.

KEY WORDS

Sexual initiation, Homoeroticism, Men, Male homosexuality, Sexual biographies, Colombia 


\section{INTRODUCCIÓN}

La iniciación sexual ha sido uno de los temas principales en el acercamiento a la sexualidad juvenil, por medio de la cual se presume el conocimiento de los comportamientos y las actitudes de los jóvenes frente a su cuerpo y su sexualidad. Según Bozon (1998), el interés por comprender el debut sexual se remonta a la década de 1990 como consecuencia de la política sexual de la época, que partía de la evidencia que era un evento cada vez más presente en la adolescencia y por fuera del matrimonio. La iniciación sexual, además, tiene diferentes representaciones: el tránsito de la niñez a la adultez, el ingreso al mercado matrimonial o el comienzo de actitudes sanas hacia la sexualidad, premisas que generan una atención especial para su reflexión.

En términos biográficos, este evento se constituye en un punto de inflexión en la trayectoria sexual de las personas, pues es un acontecimiento que condensa una historia preliminar (transformaciones biológicas y construcción del deseo) y que representa una disposición frente a la vida sexual posterior (Stern et al., 2003; Figueiredo de Sousa y Gomes, 2009).

Sumado a lo anterior, la iniciación sexual se ha convertido en un indicador para evaluar las políticas públicas sobre sexualidad juvenil promovidas por los Estados y organismos internacionales. Esta premisa se remonta a la relevancia social y política dirigida a la juventud en los años ochenta del siglo pasado, asunto que promovió una agenda especial para los jóvenes en la política sexual impartida una década después en las conferencias mundiales [Cairo, 1994 y
Beijing, 1995]. Se crearon, entonces, una serie de indicadores que trazaron un diagnóstico y una evaluación del comportamiento sexual de esta población; entre ellos, se encuentra la indagación de la entrada a la vida sexual, un evento en el cual se refleja la eficiencia de los programas gubernamentales dirigidos a la prevención del embarazo adolescente, las ITS y el VIH/SIDA. Sin embargo, la agenda se dirigió principalmente hacia las mujeres debido a la centralidad en la salud reproductiva, quedando los varones en un segundo plano.

Por otra parte, los estudios sobre sexualidad juvenil se han concentrado en las relaciones heterosexuales, dejando de lado un amplio escenario sexual como, por ejemplo, el homoerotismo, asunto que podría catalogarse como un vacío mayúsculo para la academia y la agenda gubernamental. Además, este vacío investigativo capta más importancia ahora que Latinoamérica está inmersa en un proceso de reconocimiento político y social de la diversidad sexual, que si bien se concentra en unas demandas de carácter jurídico, también implica otros tipos de acercamientos a su configuración social, entre ellos, la sexualidad.

En particular, el acercamiento al inicio de la vida sexual homoerótica en varones cobra relevancia debido a otras razones, entre ellas, a la aceptación de que la entrada a la vida sexual por parte de los hombres está inmersa en diversos juegos en el que pueden estar involucrados compañeros de su mismo sexo (Gubert y Madureira, 2009). Asimismo es importante debido a la configuración del orden sexual en Latinoamérica que, según varios autores (Almaguer, 1995; Schifter, 1998; Parker, 2000), incita 
a los varones a comprobar su hombría por medio de su desempeño sexual no sólo con mujeres, sino incluso con hombres, sin que con ello se comprometa su heterosexualidad.

A diferencia de las mujeres, la entrada de la vida sexual de los varones tiene sus particularidades (Amuchástegui, 2000). Las pautas fundamentales para la comprensión de su iniciación sexual son: la comprobación de la masculinidad (Szasz y Lerner, 1998; Geldstein y Schufer, 2002; Rojas y Castrejón, 2007), la presión de pares (Trujillo y Barrera, 2002) y el bajo involucramiento emocional (Necchi y Schufer, 1999; Juárez, 2009). También se ha recalcado que los hombres tienen mayor responsabilidad de cumplir con la iniciación sexual bajo unos patrones de edad, contexto y desempeño sexual, antecedentes que instauran diferencias en torno a la motivación, la exclusividad, la atracción, el deseo y las fantasías asumidas por los varones en torno al debut sexual.

En términos de prácticas, los estudios advierten que los jóvenes identifican la iniciación sexual con tres sucesos principales: masturbación, rompimiento del prepucio y sexualidad coital, sea hetero u homoerótica (Ayús y Tuñón, 2007; Da Silva Brêtas et al., 2011). En otras investigaciones se han identificado tres sucesos más: los intentos de penetración, el faje y los juegos sexuales entre pares, que implican una exploración del cuerpo de lo(s) otro(s) y donde es posible la realización de algunas de las anteriores prácticas (Gallego, 2010).

Los antecedentes sobre la incursión a la vida homoerótica son pocos $\mathrm{y}$, en general, revelan que los patrones de iniciación sexual entre personas del mismo sexo no son muy distintos a los heterosexuales (Cáceres, 1999; USAID, 2007; Gallego, 2010). Respecto a la iniciación entre varones, se ha estimado que los 16 años son la edad mediana de la primera relación sexual. Cuando se observa la velocidad con la cual los jóvenes de hoy cumplen su iniciación sexual, se nota una aceleración considerable entre las cohortes más jóvenes con respecto a las adultas. Otros resultados destacan que la primera experiencia homoerótica se presenta en las redes construidas en la escuela, en los grupos de vecinos y entre parientes. La vivienda familiar de alguno de los participantes prevalece como el escenario donde transcurre el encuentro. Finalmente, se subraya un bajo uso del condón en el debut sexual entre varones.

El presente artículo es una apuesta por enriquecer los antecedentes sobre el tema, por ello se presentan los patrones de iniciación sexual de un grupo de 401 hombres con prácticas homoeróticas de la región centro occidente colombiana (véase mapa 1, pág. siguiente). El estudio no asocia exclusivamente la iniciación sexual con la penetración anal que ha sido el enfoque predominante en los estudios sobre sexualidad masculina desde una perspectiva epidemiológica y de salud pública; en nuestro caso, se partió de la valoración subjetiva que los encuestados consideraron como su primera experiencia sexual con otro hombre, esto incluye la masturbación mutua, los juegos sexuales o lo que en Colombia se conoce como bluyineo, ${ }^{1}$ un tipo de

1 El "bluyineo" hace referencia al acercamiento sexual intenso en el que por condiciones del momento generalmente no se quita la ropa. La expresión viene de la palabra "bluejeans", que es una derivación regional para designar un tipo de pantalón. 
práctica sexual donde se da un acercamiento intenso sin desnudarse. De igual manera, el estudio indaga por la construcción del deseo homoerótico previo a la primera experiencia sexual. Posteriormente, se explora si el debut fue homosexual o heterosexual, pues algunas referencias revelan que las personas con prácticas homoeróticas no tienen una exclusividad en cuanto el sexo de sus parejas, aunque existe una diferencia marcada ya que las mujeres tienen más compañeros del sexo opuesto (Sandfort, 1998; Heilborn y Cabral, 2006). Luego, se rastrea en detalle el evento: edad de los implicados, tipo de relación que tenía con la pareja, lugar donde ocurrió, el proceso por el cual conoció a la pareja sexual, la iniciativa, las prácticas sexuales que se tuvieron y finalmente si hubo protección o no (uso del condón). Estos cuestionamientos permitieron estudiar a fondo la configuración de la iniciación sexual de los encuestados, centrándonos en asuntos que dieran cuenta de las relaciones de poder implícitas en el evento a partir de las diferencias generacionales.

\section{Mapa 1. Colombia y Región Cafetera} donde se adelantó el estudio

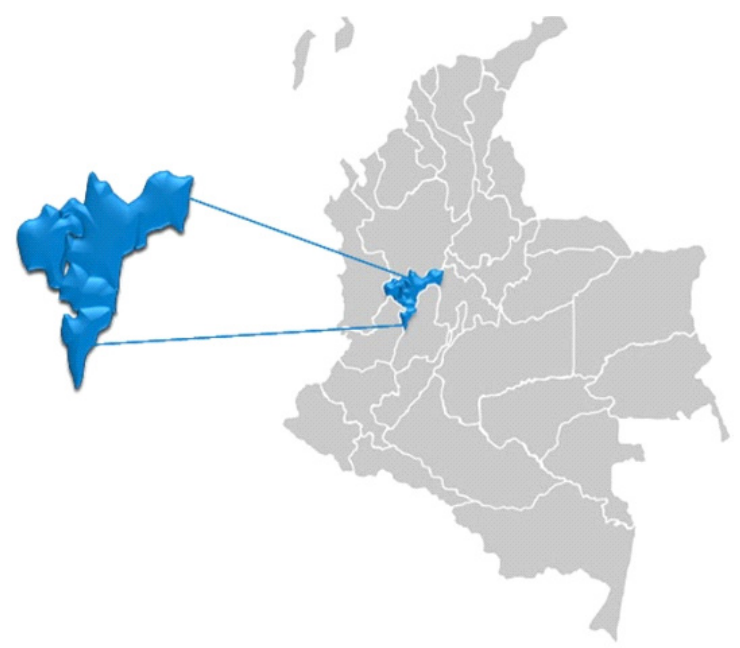

\section{MATERIAL Y MÉTODOS}

La Universidad de Caldas con apoyo financiero de COLCIENCIAS adelantó el proyecto de investigación Biografías socio-sexuales en Varones y Mujeres con prácticas homoeróticas en el Eje Cafetero colombiano. La recolección de información se realizó, mediante la aplicación de una encuesta biográfica o retrospectiva, durante cinco meses del año 2012; existen versiones del cuestionario para hombres como para mujeres, con la misma batería de preguntas. En total se entrevistaron 401 hombres, que constituyen una muestra no probabilística.

Para la recolección de la información se utilizó como método principal el RDS (Respondent Driven Sampling), que permitió captar el 68\% de las encuestas; también se utilizó la bola de nieve (16.1\%) y abordaje directo en sitios conocidos de ligue o de socialización gay-homosexual (15.9\%). Estos tres métodos de recolección de información tienen en común el uso de cadenas de referenciados. El trabajo de campo se adelantó en las áreas metropolitanas del Eje Cafetero: Manizales (28.7\%), Pereira (24.7\%), Armenia (20.3\%) y Cartago (26.2\%).

Para el análisis de la información se definieron cohortes de nacimiento que constituye el principio central del análisis del enfoque biográfico o de curso de vida; la primera cohorte la conforman las hombres nacidos antes de 1970, es decir, mayores de 41 años (33.2\%). La segunda agrupa los nacidos entre 1971 y 1983 cuyas edades están entre los 28 y 40 años (30.9\%); y la tercera abarca los nacidos entre 1984 y 1993 con edades entre los 18 y 27 años (35.9\%). 
A todos los hombres que participaron en la investigación se les informó de manera previa a la encuesta los propósitos y la utilidad de la información, la confidencialidad y el tratamiento estadístico que se le daría a los datos, todo dentro del marco del consentimiento informado. Este protocolo fue aprobado por el Comité de Ética de la Universidad de Caldas.

Para el manejo estadístico de la información se usó el paquete estadístico SPSS versión 20. El cálculo de la duración mediana de la edad de ocurrencia de la primera relación sexual y la intensidad del mismo se obtuvo a partir de tablas de sobrevivencia.

\section{LA EMERGENCIA DEL DESEO EN VARONES CON PRÁCTICAS HOMOERÓTICAS}

La indagación biográfica partió de la construcción del deseo anterior a la primera relación sexual, conteniendo preguntas sobre el momento del curso de vida en que el encuestado se sintió atraído por alguien de su mismo sexo, quién era la persona, si llegó a tener relaciones sexuales con ella y cuáles fueron las reacciones personales ante la atracción. La construcción del deseo sexual es un proceso complejo que inicia desde edades muy tempranas y pone en juego los dispositivos de socialización impartidos, principalmente, por la familia y la escuela, y sustentados en un orden sexual específico. Es por lo anterior que experimentar deseos distintos al mandato puede generar ansiedad o confusión en algunas personas, aunque para otras puede resultar una experiencia sin mayores contratiempos. Lo importante es considerar que la entrada al homoerotismo - aun cuando no sea en la práctica-, es un proceso con complicaciones y obstáculos, situación que es distinta en la inserción a la vida heterosexual.

En el cuadro 1 (pág. siguiente) se aprecia el momento en que los encuestados se sintieron atraídos por una persona del mismo sexo, reiterándose que éste ocurre a edades muy tempranas, durante la niñez o la adolescencia. Respecto a las diferencias por cohorte, los hombres mayores de 40 años tardaron más tiempo en reconocer su atracción homosexual, mientras que los más jóvenes presentan una tendencia a la atracción a edades más tempranas $(64.6 \%)$. Este tipo de procesos de reconocimiento a vidas sexuales distintas a la heterosexual son de reciente aparición en la cultura occidental y permiten la emergencia de nuevas subjetividades en torno al deseo sexual; los hombres encuestados de mayor edad, tal vez por la situación descrita, no contaban con herramientas discursivas que les permitiera legitimar su situación y reconocerse en una sexualidad distinta a la hegemónica. Para las generaciones más jóvenes, que nacieron en medio del movimiento de liberación homosexual y de un influjo mediático sobre este asunto, les ha permitido enunciar y admitir su deseo sexual hacia personas del mismo sexo desde edades tempranas, sin tanta estigmatización social como en épocas anteriores.

Respecto al vínculo de la persona que despertó la atracción, no hay diferencias representativas por cohorte, predominando un amigo o compañero de estudio en tres de cuatro encuestados; un familiar/pariente aparece como opción en el $12.8 \%$ de los casos, seguido de un maestro/profesor. La familia y la escuela como escenarios que propiciaron la emergencia del deseo sexual es entendible por el papel que 
Cuadro 1. Emergencia del deseo en varones con prácticas homoeróticas por cohorte de nacimiento en (\%). Eje Cafetero, 2012

\begin{tabular}{|c|c|c|c|c|}
\hline & $\begin{array}{c}\text { Total } \\
(\mathrm{N}=401)\end{array}$ & $\begin{array}{c}\text { Antes de } \\
1970 \\
(n=133)\end{array}$ & $\begin{array}{c}\text { Entre } \\
1971-1983 \\
(\mathrm{n}=124)\end{array}$ & $\begin{array}{c}\text { Entre } \\
1984-1993 \\
(\mathrm{n}=144)\end{array}$ \\
\hline \multicolumn{5}{|c|}{$\begin{array}{l}\text { ¿En qué momento de su vida se sintió } \\
\text { atraído por alguien de su mismo sexo? }\end{array}$} \\
\hline Niñez (antes de los 12 años) & 59,4 & 56,4 & 56,5 & 64,6 \\
\hline Adolescencia (13-17 años) & 31,9 & 32,3 & 29,8 & 33,3 \\
\hline Juventud (18-26 años) & 8,2 & 10,5 & 12,9 & 2,1 \\
\hline Adultez (más de los 27 años) & 0,5 & 0,8 & 0,8 & 0 \\
\hline \multicolumn{5}{|l|}{ ¿Quién era esa persona? } \\
\hline Amigo & 43,8 & 39,4 & 44,4 & 47,2 \\
\hline Compañero de estudio & 27,0 & 25,0 & 28,2 & 27,8 \\
\hline Familiar/pariente & 12,8 & 15,2 & 10,5 & 12,5 \\
\hline Maestro-profesor & 3,5 & 5,3 & 1,6 & 3,5 \\
\hline Otra persona & 12,9 & 15,1 & 15,3 & 9,0 \\
\hline \multicolumn{5}{|c|}{ ¿Cómo se sintió en ese momento? } \\
\hline Apenado & 6,7 & 6,8 & 5,6 & 7,6 \\
\hline Emocionado & 24,4 & 30,1 & 24,2 & 19,4 \\
\hline Culpable & 6,5 & 7,5 & 8,1 & 4,2 \\
\hline Confundido & 36,9 & 30,8 & 33,9 & 45,1 \\
\hline Otro sentimiento & 25,5 & 24,8 & 28,2 & 23,7 \\
\hline \multicolumn{5}{|c|}{$\begin{array}{l}\text { ¿Con esta pesona, llegó a tener } \\
\text { relaciones sexuales? }\end{array}$} \\
\hline$S i$ & 57,9 & 66,2 & 55,6 & 52,1 \\
\hline
\end{tabular}

Fuente: Construcción propia a partir de la encuesta Biografias sexuales... en el Eje Cafetero

cumplen estas dos instituciones en los primeros años de vida de los individuos: ellas comparten la tarea de desarrollar los primeros procesos de socialización en los que se prepara al sujeto para afrontar/interiorizar una estructura social determinada, incluyendo la heterosexualidad normativa. La casa, el vecindario y la escuela terminan siendo las coordenadas donde se mueve la construcción del deseo sexual, el campo donde se despliega la sexualidad como práctica social compleja, sus topos.

Acerca de los sentimientos que vivieron los varones en el momento de sentirse atraídos por una persona del mismo sexo, las respuestas son amplias y variadas; "la confusión" es la sensación más representativa $(36.9 \%)$. Llegar a sentir 
atracción homosexual se constituye en una señal de que "algo se está haciendo mal", una afrenta tanto para la vida social como individual del sujeto. Por otro lado, uno de cuatro encuestados se sintió emocionado al experimentar deseo por alguien del mismo sexo, mientras que el sentirse culpable es una experiencia que tiende a perder importancia en las generaciones más jóvenes.

En términos de prácticas sexuales asociadas al deseo, se encuentra que uno de dos entrevistados llegó a tener relaciones sexuales con la persona por quien sentía atracción, situación vivida en un porcentaje mayor en la cohorte adulta. Los datos parecen indicar que entre los más jóvenes existe una tendencia a desvincular el deseo del debut sexual y postergar la primera relación homoerótica para otro momento del curso de vida.

\section{LA PRIMERA RELACIÓN SEXUAL HOMOERÓTICA:}

\section{DESPLAZAMIENTOS Y (DIS)CONTINUIDADES}

La iniciación sexual de las personas que tienen prácticas homoeróticas no siempre está relacionada con una persona del mismo sexo, lo que da cuenta de los tránsitos por los que pasa la sexualidad a lo largo de la vida e indica que no existen rutas genéricamente establecidas. En términos generales, el $71 \%$ de los encuestados tuvo su primera experiencia sexual con otro hombre, en los jóvenes este porcentaje tiende a subir, mostrando un patrón de mayor exclusividad sexual. Al revisar el conjunto de las interacciones sexuales se observa que el $58 \%$ de los hombres había tenido en su curso biográfico relaciones sexuales con hombres y con mujeres, existiendo una disminución entre cohortes en la iniciación sexual homoerótica antes de la heterosexual, pasando del $56.1 \%$ al $39.2 \%$ en los más jóvenes (véase cuadro 2, pág. siguiente). También se presenta una tendencia a la simultaneidad del evento entre los más jóvenes (véase cuadro 3 , pág. siguiente), en un ejercicio de exploración, búsqueda y construcción de la sexualidad juvenil que puede indicar nuevos patrones sexuales y la emergencia de subjetividades que no agotan sus prácticas eróticas en un solo sexo. Un dato que confirma el hallazgo anterior es la estimación de la edad de inicio de la vida sexual, la cual permanece relativamente estable entre cohortes en los 15 años y es muy cercana a la edad mediana en la cual se inician sexualmente los jóvenes en Colombia. 


\begin{tabular}{|l|c|c|c|c|}
\hline $\begin{array}{c}\text { Cuadro 2. Distribución porcentual de los encuestados según la precedencia temporal de la primera } \\
\text { relación homoerótica con respecto a la heterosexual (de los que vivieron ambos eventos), } \\
\text { por cohorte de nacimiento. Eje Cafetero, 2012 }\end{array}$ \\
\cline { 2 - 5 } Precedencia de los eventos sexuales & $\begin{array}{c}\text { Cohorte de nacimiento } \\
(\mathrm{n}=234)\end{array}$ & $\begin{array}{c}\text { Antes de } \\
1970 \\
(\mathrm{n}=89)\end{array}$ & $\begin{array}{c}\text { Entre } \\
1971-1983 \\
(\mathrm{n}=76)\end{array}$ & $\begin{array}{c}\text { Entre } \\
1984-1993 \\
(\mathrm{n}=69)\end{array}$ \\
\hline Homoerótica antes de la heterosexual & 45,3 & 56,1 & 38,2 & 39,2 \\
Heterosexual antes de la homoerótica & 45,3 & 36,0 & 53,9 & 47,8 \\
Eventos simultáneos & 9,4 & 7,9 & 7,9 & 13,0 \\
\hline
\end{tabular}

Fuente: Construcción propia a partir de la encuesta Biografías sexuales... en el Eje Cafetero

\begin{tabular}{|l|c|c|c|}
\hline \multicolumn{3}{|c|}{$\begin{array}{c}\text { Cuadro 3. Edad mediana de la primera experiencia homoerótica y } \\
\text { heterosexual por cohorte de nacimiento. Eje cafetero, } 2012\end{array}$} \\
\hline \multirow{2}{*}{ Evento sexual } & \multicolumn{3}{c|}{ Cohorte de Nacimiento } \\
\cline { 2 - 4 } & Antes de & Entre & Entre \\
& 1970 & $1971-1983$ & $1984-1993$ \\
\hline Homoerótico & $\mathbf{1 5 , 7 5}$ & $\mathbf{1 6 , 1 9}$ & $\mathbf{1 5 , 4 4}$ \\
Heterosexual & $\mathbf{1 7 , 7 9}$ & $\mathbf{1 6 , 5 9}$ & $\mathbf{1 5 , 5}$ \\
Diferencias & $-2,04$ & $-0,40$ & $-0,06$ \\
\hline
\end{tabular}

Fuente: Construcción propia a partir de la encuesta Biografías sexuales... en el Eje Cafetero

Es indispensable comprender la iniciación sexual en edades más tempranas o tardías a la edad mediana presentadas anteriormente. A partir de la información del cuadro 4 ( pág. siguiente) surge un elemento que es importante considerar en cuanto que la cuarta parte de los varones tuvieron su primera experiencia sexual antes de los 12 años, lo cual puede indicar que esta práctica social transcurre en ámbitos familiares o vecinales debido a que las redes sociales de los menores se agotan en estos contextos. En cuanto a las diferencias entre las cohortes, es significativo el hecho de que los más jóvenes tienen más velocidad en el cumplimiento del evento. Partiendo de los 14 años, edad en la que las tres generaciones presentan cifras muy similares (40\%), se destaca el hecho de que la cohorte más adulta haya tardado hasta los 23 años para llegar a un 95\% de cumplimiento, porcentaje que en los más jóvenes se alcanzó a los 18 años. 


\begin{tabular}{|c|c|c|c|}
\hline \multicolumn{3}{|c|}{$\begin{array}{c}\text { Cuadro 4. Porcentaje acumulado de varones que a la edad } \\
\text { exacta X habían tenido su primera experiencia homoerótica, } \\
\text { por cohorte de nacimiento. Eje Cafetero, } 2012\end{array}$} \\
\hline \multirow{3}{*}{ Edad } & \multicolumn{3}{|c|}{ Cohorte de Nacimiento } \\
\cline { 2 - 4 } & Antes de 1970 & Entre & Entre \\
& $(\mathrm{n}=133)$ & $1971-1983$ & $1984-1993$ \\
& & $\mathrm{n}=124)$ & $(\mathrm{n}=144)$ \\
\hline 7 & 4,0 & 7,0 & 1,0 \\
8 & 8,0 & 7,0 & 6,0 \\
9 & 12,0 & 11,0 & 8,0 \\
10 & 17,0 & 14,0 & 11,0 \\
11 & 20,0 & 18,0 & 13,0 \\
12 & 29,0 & 26,0 & 21,0 \\
13 & 35,0 & 31,0 & 27,0 \\
14 & 42,0 & 41,0 & 43,0 \\
15 & 53,0 & 48,0 & 59,0 \\
16 & 62,0 & 59,0 & 71,0 \\
17 & 69,0 & 70,0 & 81,0 \\
18 & 76,0 & 79,0 & 95,0 \\
19 & 83,0 & 83,0 & 97,0 \\
20 & 89,0 & 86,0 & 97,0 \\
21 & 92,0 & 87,0 & 99,0 \\
22 & 92,0 & 88,0 & 99,0 \\
23 & 95,0 & 88,0 & 99,0 \\
24 & 95,0 & 92,0 & 100,0 \\
\hline
\end{tabular}

Fuente: Construcción propia a partir de la encuesta Biografías sexuales... en el Eje Cafetero

\section{RECONSTRUCCIÓN DE LA PRIMERA}

\section{EXPERIENCIA HOMOERÓTICA:}

\section{ESCENARIOS Y PRÁCTICAS}

La indagación por la primera experiencia sexual también integra la aproximación al contexto situacional donde ocurrió el evento. Preguntar por estos asuntos resulta pertinente para comprender las formas y las relaciones bajo las cuales acontecen los primeros acercamientos sexuales de las personas, panorama en los que están inmersos elementos culturales y de poder que inciden en la configuración del debut sexual. De acuerdo a las estimaciones del cuadro 5 (pág. siguiente), la primera experiencia homoerótica parece ser un asunto que se lleva a cabo en ámbitos sociales cercanos, es decir, se concentra en los lazos sociales más íntimos como la familia, la amistad, los vecinos y las relaciones sentimentales (60\%). 
Cuadro 5. Distribución porcentual de los encuestados según contexto de la primera experiencia homoerótica por cohorte de nacimiento. Eje Cafetero, 2012

\begin{tabular}{|c|c|c|c|c|}
\hline \multirow[b]{2}{*}{ Tipo de vínculo con el compañero sexual } & \multicolumn{4}{|c|}{ Cohorte de nacimiento } \\
\hline & $\begin{array}{c}\text { Total } \\
(n=401)\end{array}$ & $\begin{array}{c}\text { Antes de } \\
1970 \\
(n=133)\end{array}$ & $\begin{array}{c}\text { Entre } \\
1971-1983 \\
(\mathrm{n}=124)\end{array}$ & $\begin{array}{c}\text { Entre } \\
1984-1993 \\
(\mathrm{n}=144)\end{array}$ \\
\hline Recién conocido & 13,4 & 13,5 & 15,1 & 12,0 \\
\hline Amigo/vecino/conocido & 49,4 & 47,6 & 51,3 & 49,3 \\
\hline Novio/pareja & 10,1 & 6,3 & 9,2 & 14,1 \\
\hline Familiar/pariente & 13,4 & 15,1 & 13,4 & 12,0 \\
\hline Compañero de estudio o trabajo & 10,9 & 14,3 & 7,6 & 10,6 \\
\hline Otro & 2,8 & 3,2 & 3,4 & 2,0 \\
\hline \multicolumn{5}{|l|}{ Diferencias de edad con el compañero } \\
\hline Pareja 2 o + años menor & 5,0 & 6,8 & 9,0 & 0,0 \\
\hline Diferencias <=1 año & 25,4 & 26,3 & 20,7 & 28,7 \\
\hline Pareja 2 o + años $>$, siendo ent $<15$ y pareja $>18$ & 16,6 & 16,5 & 18,2 & 15,4 \\
\hline Pareja $2 \mathrm{o}+$ años $>$, siendo ent $>=15$ y pareja $>18$ & 23,2 & 19,5 & 21,5 & 28,0 \\
\hline Pareja 2 o + años $>$, siendo ambos $<18$ & 15,4 & 15,1 & 14,9 & 16,0 \\
\hline Pareja $2 \mathrm{o}+$ años $>$, siendo ambos $>18$ & 14,4 & 15,8 & 15,7 & 11,9 \\
\hline \multicolumn{5}{|l|}{ Lugar de conocimiento de la pareja } \\
\hline En la familia & 20,2 & 19,7 & 19,0 & 21,5 \\
\hline En el barrio o vereda & 32,2 & 28,3 & 44,8 & 25,9 \\
\hline En el colegio/universidad & 22,9 & 23,6 & 17,1 & 26,7 \\
\hline En una organización o lugar de trabajo & 5,1 & 9,5 & 4,8 & 1,5 \\
\hline En la calle & 9,8 & 9,4 & 7,6 & 11,9 \\
\hline Por internet & 2,5 & 0,0 & 2,9 & 4,4 \\
\hline Otro lugar & 7,3 & 9,5 & 3,8 & 8,1 \\
\hline \multicolumn{5}{|l|}{ Quién los presentó } \\
\hline Vecinos-familiares & 36,0 & 36,7 & 37,2 & 34,5 \\
\hline Red de amigos(as) & 30,8 & 32,0 & 30,1 & 30,3 \\
\hline Autopresentación & 32,6 & 30,5 & 32,7 & 34,5 \\
\hline Otro & 0,6 & 0,8 & 0,0 & 0,7 \\
\hline \multicolumn{5}{|l|}{ Lugar donde ocurrió el encuentro } \\
\hline Casa de uno de ellos & 65,5 & 56,5 & 65,5 & 74,1 \\
\hline Casa de un amigo & 7,0 & 6,9 & 7,8 & 6,5 \\
\hline Motel/hotel & 7,3 & 9,9 & 5,2 & 6,5 \\
\hline Zona rural, cultivo o bosque & 11,1 & 20,3 & 9,4 & 3,6 \\
\hline Otro lugar & 9,1 & 6,4 & 12,1 & 9,3 \\
\hline
\end{tabular}

Fuente: Construcción propia a partir de la encuesta Biografias sexuales... en el Eje Cafetero 
Sin embargo, se advierte que para los más jóvenes los vínculos sentimentales como el noviazgo están ganando valoración, pasando de $6.3 \%$ en los más adultos a $14.1 \%$ entre los menores de 28 años. El aumento de la categoría "novio" debe enmarcarse en un contexto de posibilidad que permite a los más jóvenes reapropiarse del discurso del "noviazgo" para designar las relaciones sentimentales entre personas del mismo sexo. De igual manera, es necesario resaltar la forma en que se mantiene una regularidad en las categorías de "recién conocido" y "familiar" como mención a sujetos con los cuales se tuvo la primera experiencia sexual y que generan una alta ansiedad social, especialmente la de familiar o pariente.

La vía de conocimiento de la pareja de la primera experiencia sexual se da generalmente a través de redes interpersonales y familiares, el $75.3 \%$ de los hombres conocieron a su pareja por medio de la familia, en el barrio o en la escuela. Otro asunto importante es la aparición de internet como un medio para el conocimiento de parejas sexuales, situación que pone de manifiesto un cambio generacional, mientras los más adultos - que no contaban con este medio tecnológico en su juventud - presentan cifras nulas en esta categoría, en las generaciones más jóvenes se puede indicar la aparición de este medio como un recurso importante para el conocimiento de la pareja, vía que seguramente seguirá aumentando en las generaciones futuras.

También es importante considerar que para los más adultos las organizaciones o lugares de trabajo se constituían como contextos posibles para el conocimiento de sus parejas, situación que ha perdido peso relativo en los más jóvenes, ya que en ellos se está postergando su vida educativa, lo que hace que entren al mundo del trabajo a edades más tardías.

De acuerdo a la pregunta sobre quién le presentó a su pareja sexual, de nuevo se confirma la tesis sobre los lazos sociales cercanos como medio en donde se despliegan los primeros acercamientos sexuales. La familia y la red de amigos aparecen como puentes importantes en los encuestados de todas las generaciones. La "autopresentación" como forma de vinculación personal tiene una carga porcentual importante en las relaciones homoeróticas que son propias de la socialización y el habitus masculino.

Otro elemento que reafirma la proximidad en la primera relación sexual son los datos del lugar donde ocurrió ésta; la casa de uno de los implicados (encuestado o pareja) constituyó el escenario donde el $65.5 \%$ de los hombres tuvo su primera experiencia homoerótica, con un ligero aumento entre cohortes. Otros lugares como moteles o zonas al aire libre (zonas rurales) ya no tienen la misma significación para los más jóvenes. 


\begin{tabular}{|c|c|c|c|c|c|c|c|}
\hline \multicolumn{8}{|c|}{$\begin{array}{l}\text { Cuadro 6. Distribución porcentual de los encuestados según contexto y práctica sexual realizada durante la } \\
\text { primera experiencia homoerótica, según diferencias de edad con respecto a la pareja. Eje Cafetero, } 2012\end{array}$} \\
\hline HOMBRES & \multicolumn{7}{|c|}{ DIFERENCIAS DE EDAD EN LA PRIMERA EXPERIENCIA HOMOERÓTICA } \\
\hline $\begin{array}{l}\text { Tipo de vínculo con el compañero } \\
\text { sexual }\end{array}$ & $\begin{array}{c}\text { Total } \\
(n=401)\end{array}$ & $\begin{array}{c}\text { Pareja } 20 \\
+ \text { años } \\
\text { menor } \\
(\mathbf{n}=\mathbf{2 0})\end{array}$ & $\begin{array}{c}\text { Diferencias } \\
<=1 \text { año } \\
(n=102)\end{array}$ & $\begin{array}{l}\text { Pareja } 20+ \\
\text { años }>, \\
\text { siendo ent } \\
<15 \text { y pareja } \\
>18(n=66)\end{array}$ & $\begin{array}{c}\text { Pareja } 2 \text { o } \\
\text { años }> \\
\text { siendo ent } \\
>=15 \text { y } \\
\text { pareja }>18 \\
(n=93)\end{array}$ & $\begin{array}{c}\text { Pareja } 20+ \\
\text { años }>, \\
\text { siendo } \\
\text { ambos }<18 \\
(\mathrm{n}=62)\end{array}$ & $\begin{array}{c}\text { Pareja } 2 \text { o+ } \\
\text { años }> \\
\text { siendo } \\
\text { ambos }>18 \\
(\mathrm{n}=\mathbf{5 8})\end{array}$ \\
\hline Recién conocido & 13,4 & 20,0 & 4,0 & 21,3 & 15,6 & 1,7 & 29,1 \\
\hline Amigo/vecino/conocido & 49,4 & 65,0 & 51,5 & 47,5 & 47,8 & 51,7 & 41,8 \\
\hline Novio/pareja & 10,1 & 5,0 & 11,9 & 4,9 & 17,8 & 5,0 & 7,3 \\
\hline Familiar/pariente & 13,4 & 5,0 & 9,9 & 19,7 & 10,0 & 30,0 & 3,6 \\
\hline Compañero estudio o trabajo & 10,9 & 5,0 & 22,7 & 0,0 & 4,4 & 11,6 & 12,7 \\
\hline Otro & 2,8 & 0,0 & 0,0 & 6,6 & 4,4 & 0,0 & 5,5 \\
\hline \multicolumn{8}{|l|}{ Iniciativa para la relación sexual } \\
\hline Entrevistado & 13,9 & 25,0 & 25,0 & 9,1 & 9,9 & 13,1 & 3,5 \\
\hline Pareja & 60,8 & 45,0 & 44,0 & 75,8 & 65,9 & 63,9 & 66,7 \\
\hline Iniciativa mutua & 25,3 & 30,0 & 31,0 & 15,1 & 24,2 & 23,0 & 29,8 \\
\hline \multicolumn{8}{|l|}{ Práctica sexual } \\
\hline Penetré y/o me hicieron sexo oral & 21,8 & 55,0 & 23,0 & 15,2 & 23,1 & 8,2 & 28,6 \\
\hline Me penetraron e/o hice sexo oral & 37,4 & 20,0 & 19,0 & 56,1 & 52,7 & 32,8 & 33,9 \\
\hline Penetración y/o sexo oral mutuo & 18,5 & 5,0 & 27,0 & 10,6 & 17,6 & 23,0 & 14,3 \\
\hline Solamente masturbación mutua & 10,4 & 15,0 & 16,0 & 7,6 & 4,4 & 11,5 & 10,7 \\
\hline Manoseo, blujiniada, calentada & 11,9 & 5,0 & 15,0 & 10,5 & 2,2 & 24,5 & 12,5 \\
\hline
\end{tabular}

Fuente: Construcción propia a partir de la encuesta Biografias sexuales... en el Eie Cafetero

Un asunto importante en la reconstrucción contextual de la primera experiencia sexual se relaciona con las diferencias de edad entre el encuestado y su pareja, en cuanto que es un elemento que señala diferencias de poder entre los implicados. Estudios como el de Gallego (2010) señalan que estas diferencias etáreas tienen relación con la iniciativa para tener el acercamiento sexual, el tipo de prácticas sexuales que mantuvieron y los vínculos construidos con la pareja. A partir de las diferencias etáreas entre el encuestado y su pareja, se construyeron seis categorías - véase cuadro 5 y 6 . De entrada, se aprecia la preeminencia de relaciones con hombres mayores dos o más años (70\%), los casos donde el encuestado era menor de 14 años y la pareja mayor de edad es una manifestación clara de abuso sexual (16.6\% en hombres).

De acuerdo con los datos contenidos en el cuadro 6, el tipo de vínculo en la primera experiencia sexual cambia según las diferencias de edad. La categoría "recién conocido" prima entre aquellos varones cuyas diferencias de edad eran mayores de dos años, siendo ambos mayores de 
edad (29.1\%), seguida por la categoría en donde el encuestado era menor de 15 años y su pareja mayor de edad (21.3\%) y, por último, cuando la pareja era dos o más años menor que éste (20\%).

En los casos donde prevalece la misma edad, la relación de amistad, vecino o conocido es el vínculo con más peso porcentual (51.5\%), seguido por el compañero de estudio o trabajo (22.7\%). Esta situación es comprensible a partir de las relaciones horizontales que se construyen en las redes sociales más cercanas, como la escuela y la vecindad, en donde la socialización se desarrolla con pares etáreos. Con relación al noviazgo, un lazo social que permite la iniciación sexual entre varones en contextos de diferencias de edad, es un vínculo que presenta su mayor porcentaje cuando la pareja es mayor de edad y el encuestado es menor de quince años (17.8\%), de modo que el noviazgo se constituye en la entrada a la vida sexual en contextos de disparidad etárea.

Las relaciones familiares aparecen como vínculo durante la primera relación sexual cuando la pareja es mayor dos o más años, siendo ambos menores de edad (30\%) y cuando la pareja es dos o más años mayor, siendo el encuestado menor de quince años y la pareja mayor de dieciocho. Este tipo de vínculo presenta su tasa más baja cuando ambos eran mayores de dieciocho años o cuando el encuestado era la persona mayor de la relación, lo que permite comprender que el contexto familiar es un escenario en el que la minoría de edad respecto a la pareja parece ser un aspecto fundamental para incentivar las primeras experiencias sexuales homoeróticas entre varones.

Las diferencias de edad entre los involucrados en las primeras relaciones sexuales tienen una alta influencia en la toma de decisión sobre el encuentro erótico, y generalmente es la persona mayor quien toma la iniciativa. Por ejemplo, las cifras superan el $60 \%$ en todos los casos en que la pareja es mayor dos o más años. De manera que las diferencias de edad son un factor determinante que denota poder de decisión en los contextos de las primeras relaciones sexuales, más aún en el campo del homoerotismo donde la entrada a la sexualidad es difícil de afrontar. Además, hay que tener presente que, aunque era la primera vez para el encuestado, tal vez no lo era para su pareja, que contaba ya con más recursos simbólicos para enfrentar este tipo de encuentros eróticos.

De igual manera, el poder que brinda la edad frente a la pareja dispone, organiza y jerarquiza las prácticas sexuales realizadas en la primera experiencia sexual. Generalmente, los varones mayores fueron quienes penetraron y a quienes les realizaron sexo oral; los casos de reciprocidad en estas prácticas se presentan cuando ambos tenían la misma edad o cuando había una diferencia de dos o más años a favor del encuestado. Entonces, la edad es un indicador de los recursos simbólicos que se recrean durante la primera relación sexual, y por medio de la cual se cimientan relaciones de poder en las que están en juego las nociones de masculinidad y feminidad de quienes están inmersos en el encuentro sexual. Tanto es así, que otras prácticas que no implican la penetración o el sexo oral presentan sus más altos porcentajes cuando los dos hombres tenían la misma edad o cuando los dos eran menores de dieciocho años, edades en las cuales hay una 
mayor búsqueda de la experimentación sexual, sin involucrar directamente nociones de poder. En este sentido, cuando el encuentro es entre dos hombres de la misma edad, hay mayor capacidad de negociación del deseo y de las prácticas dentro del encuentro sexual.

Estos cambios revelan varios asuntos, el más significativo es el traslape al campo del homoerotismo del discurso "normalizador" que asocia el debut sexual con la penetración. Este argumento reafirma la heteronormatividad y una posición ambivalente sobre el riesgo asociado a la penetración, y descarta otras prácticas sexuales no insertivas/receptivas como campo posible del erotismo entre varones. En síntesis, de la lectura del cuadro 6 se destacan varios asuntos:

- Cuando la pareja es dos o más años mayor que el entrevistado y éste es menor de edad, el varón mayor penetró al menor y/o el menor hizo sexo oral al mayor.

- Cuando el entrevistado fue el varón mayor y su pareja menor tiende a suceder la situación contraria.

- En los casos donde tanto el entrevistado como su pareja eran de la misma edad o persistían diferencias de dos o más años en favor de la pareja, pero ambos eran menores o mayores de edad, existe un encuentro sexual que podríamos denominar "democrático" donde son recíprocas las penetraciones, el sexo oral y la masturbación mutua.
Finalmente, dentro de este apartado se presenta el uso o no del condón durante la primera experiencia sexual homoerótica entre varones. El uso del condón es un asunto que tiene una alta importancia dentro de las nociones de la salud sexual y reproductiva después de la aparición de la epidemia del VIH/SIDA, a partir de la cual la promoción del uso del preservativo es un asunto primordial de las campañas dirigidas a sectores de varones con prácticas homoeróticas. Sin embargo, la información contenida en el gráfico 1 (pág. siguiente) desestima el alcance de este discurso institucional sobre el uso del condón como una práctica recomendada para el sexo entre varones.

De acuerdo con la información, existe una tendencia al aumento del uso del condón entre cohortes de encuestados, llegando al $40 \%$ en la cohorte más joven; hay que tener en cuenta que en la época en que la cohorte adulta e intermedia experimentó su primera relación sexual el uso del condón no estaba muy difundido y ésta puede ser una explicación para las bajas tasas halladas. No obstante y como lo señala la teoría de la interacción sexual, ciertas prácticas sexuales condicionan otras en la biografía sexual, de ahí que no resulte extraño cómo en el Eje Cafetero colombiano el uso del condón no supere el $30 \%$ en los encuentros homoeróticos (Minsalud-UNFPA, 2011), porcentaje que, además, se reduce en el marco de una relación de pareja estable.

Por último, es necesario vincular el uso del condón con el tipo de práctica sexual ocurrida durante la primera experiencia homoerótica. De acuerdo con el cuadro 6, el $77.7 \%$ de los entrevistados tuvieron prácticas de riesgo (penetración-sexo oral), un porcentaje elevado que denota la poca 


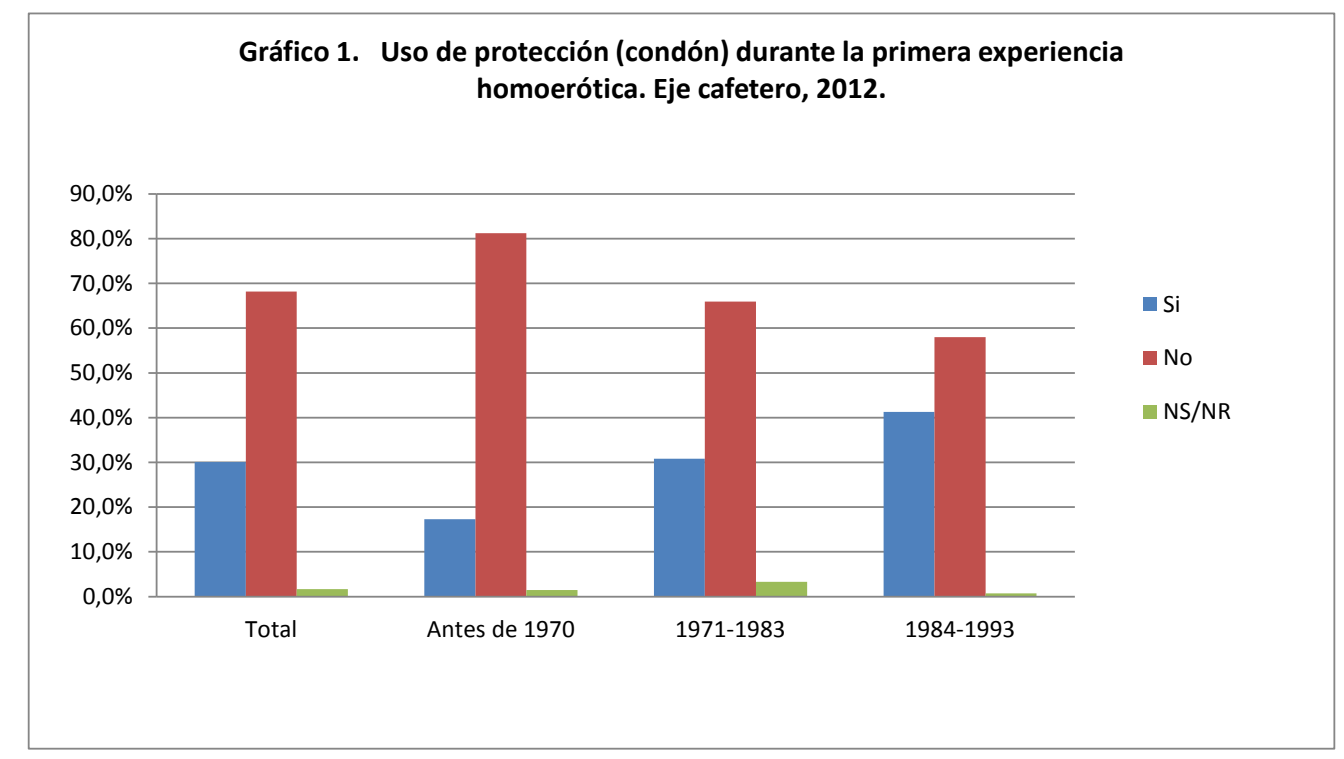

Fuente: construcción propia a partir de la Encuesta Biografias sexuales...en el Eje Cafetero

representatividad del uso del condón entre los varones encuestados. A pesar de que existe una difusión sobre las prácticas de protección por parte de organismos estatales y de agencias de cooperación nacionales e internacionales, las cifras demuestran que éste un asunto que no ha permeado lo suficiente en el contexto del homoerostimo entre varones, por lo menos en su debut sexual.

\section{APORTES PARA LA DISCUSIÓN SOBRE LA INICIACIÓN SEXUAL HOMOERÓTICA ENTRE VARONES}

A partir de los hallazgos señalados a lo largo del artículo se identifican cinco asuntos nodales sobre los que se plantea la discusión de la iniciación sexual en el campo del homoerotismo entre varones: la construcción del deseo, los cambios en los patrones de iniciación por cohorte, la familia y la escuela como escenarios centrales, la relación con trayectorias heterosexuales y, finalmente, las relaciones de poder traducidas en la feminización del cuerpo del otro.

Algunos estudios han señalado que se debe replantear la iniciación sexual como hecho fundante de la vida sexual de los jóvenes, pues es una postura que deja de lado la construcción del deseo anterior a dicho evento (Vilela y Schor, 2007). Tal vez este supuesto obedezca a varias razones: primero, a la invisibilidad de la sexualidad en la niñez, asunto que aunque ha sido rebatido por el psicoanálisis todavía es controversial para algunos sectores sociales. Segundo, por la centralidad que toma lo coital en los programas de salud sexual y reproductiva, en donde lo único que importa son las conductas de riesgo asociadas a salud/enfermedad y no las subjetividades implícitas en el encuentro sexual. Y por último, a la relación directa de la sexualidad con el acto coital, analogía que empobrece la comprensión de 
otras prácticas implícitas en el desarrollo sexual de los jóvenes.

Al cambiar el punto de referencia emergen diversas aristas por las cuales se puede lograr una mejor comprensión de la sexualidad adolescente. Temas como la experimentación a través del juego, la masturbación, el autodescubrimiento o las primeras atracciones eróticas son algunos preámbulos realizados por los jóvenes (Fuller, 2001). En este sentido, la apuesta por profundizar en la construcción del deseo resulta ser una provocación para enriquecer el análisis y promover políticas de intervención más acertadas. En este caso, el estudio permite comprobar que la atracción sexual emerge a edades tempranas y en los contextos sociales más cercanos (familia y escuela). Sin embargo, la entrada al homoerotismo es un proceso complejo, eso lo demuestran las cifras sobre los sentimientos experimentados por los encuestados al momento de advertir el deseo por una persona del mismo sexo.

Los cambios generacionales en los patrones de la construcción del deseo y de la iniciación sexual son evidentes y se constituyen en un hallazgo fundamental del estudio. Sin duda, la transformación del orden sexual y la aparición de los discursos en defensa de la diversidad sexual en las últimas décadas, han sido plataformas que han impactado en la configuración social del homoerotismo (Argüello, 2013). De ser un asunto que estaba inmerso en una trama de silencios y refugios simbólicos, pasó a convertirse en un tema de la palestra pública. Esta transformación no sólo ha hecho mella en los avances políticos registrados en los países del hemisferio (Díez, 2015), sino que también interviene en las trayectorias sexuales de aquellos que tienen prácticas homoeróticas; además, crea condiciones para el surgimiento de nuevas subjetividades en torno a la vida homosexual que desestiman el poderío de la heterosexualidad normativa.

Algunos ejemplos son el aceleramiento de la iniciación sexual en los más jóvenes respecto a las cohortes más adultas y las diferencias en la configuración del primer encuentro sexual en cuanto a compañero, edades y lugares. Un hallazgo a destacar es la tendencia a relacionarse con personas de la misma edad, que podría catalogarse como una democratización de la sexualidad homoerótica, pues es un factor que evita las figuras de poder que se tejen en las relaciones con diferencias de edad marcadas.

Otro punto es la aparición de la familia y la escuela como escenarios donde emerge el deseo sexual. El hecho de que la familia aparezca como un contexto en el cual se presentan las primeras manifestaciones sexuales puede resultar alarmante; sin embargo, este tipo de hallazgos ayudan a desmantelar la asexualidad en la que ha estado sumida la familia como institución, premisa que ha sido refutada por los datos sobre abuso sexual infantil delatados por diversos organismos que indican un involucramiento de miembros de la familia en este tipo de delito (Instituto Nacional de Medicina Legal y Ciencias Forenses, 2014). En el presente estudio, el debut sexual con un familiar prevalece en las iniciaciones más tempranas y con una persona mayor, lo que podría ser un indicio sobre casos de abuso. En cuanto a la escuela, los resultados son una señal para promover una cultura institucional inclusiva en asuntos sexuales y de diversidad sexual, más ahora que el ámbito escolar está afectado por el 
fenómeno del bullying. No obstante, se insinúa que estos dos contextos están perdiendo jurisdicción en la sexualidad de la niñez y la juventud, pues existen otras herramientas, como los mass media y el grupo de pares, que están interviniendo ampliamente en los descubrimientos sexuales de los jóvenes.

La iniciación sexual de los hombres que tienen prácticas homoeróticas no siempre está relacionada con una persona del mismo sexo. Datos como: una cuarta parte de los participantes iniciaron su vida sexual con una mujer, más de la mitad tienen en su curso biográfico relaciones sexuales con hombres y con mujeres, y la tendencia a la simultaneidad del evento entre los más jóvenes, indican la pertinencia de reconocer las tramas por las cuales transcurren las trayectorias sexuales homoeróticas. Estas rutas permiten realizar varios análisis: uno de ellos es recordar que el homoerotismo no implica asuntos de identidad sexual, sino que hace énfasis en la práctica; por lo tanto, aunque hayan varones que realizan procesos de autodefinición que denotan su homoerotismo, se incluyen otras posibilidades como el ocultamiento, la negación y la represión (Núñez Noriega, 2000). También, son una advertencia para los programas de salud sexual y reproductiva, ya que entre líneas presumen una exclusividad sexual de sus destinatarios, tanto así que muchas campañas se dirigen a población heterosexual o homosexual en específico, olvidando las fisuras y desplazamientos que existen en las trayectorias sexuales de los sujetos.

Para terminar, el homoerotismo entre varones está configurado por tramas de poder definidas por la edad, la etnicidad y la clase social de los implicados (Fuller, 2001; Gallego, 2011); en otras palabras, es un evento que debe leerse en clave interseccional (Hill Collins, 1998). Estas circunstancias evocan que la construcción de la masculinidad está infundida por la búsqueda de poder, demanda que hace uso de cualquier jerarquía social que le sirva de fundamento para justificar su arbitrariedad. El ámbito sexual es uno de los campos propicios para la puesta en marcha de estos órdenes de género, pues en medio de éste se concreta la dominación física o simbólica entre los cuerpos.

Los varones, aun en espacios liminales como en el homoerotismo, están incitados a comprobar su hombría, pues es un recurso que le brinda seguridad a su identidad genérica. En los encuentros sexuales entre varones irrumpen las expectativas de masculinidad de cada uno de los implicados, aun siendo personas con procesos identitarios no heterosexuales (gay, bisexual, trans); por ello, las prácticas sexuales se convierten en signos de demostración o transgresión de sus representaciones de género. En medio del homoerotismo, entre varones se simbolizan los cuerpos de acuerdo a los arreglos que se concretan en el encuentro sexual. Una de las operaciones más reiteradas es la feminización de su compañero sexual, asunto que se define según los roles desempeñados en prácticas sexuales como el sexo oral y la penetración.

De acuerdo a los resultados presentados páginas atrás, se destaca la edad como un factor fundamental para los arreglos del primer encuentro sexual entre varones. Las diferencias de edad repercutieron en quién toma la iniciativa, 
en las prácticas sexuales que se mantuvieron $\mathrm{y}$ en el tipo de vínculo construido con la pareja. Generalmente, los hombres mayores son los que detentan el mando de las decisiones y quienes cumplen un rol activo en la relación sexual (en los casos donde hay penetración). Mientras que la capacidad de negociación o de manifestación del deseo de satisfacción mutua se concreta especialmente cuando el encuentro homoerótico se da con un hombre de la misma edad.

En este sentido, existe una relación entre juventud y feminización en las relaciones homoeróticas entre varones, asunto que se puede comprender, según List (2005), por la jerarquía de edad asumida en la masculinidad, en la que el niño y el adolescente no se consideran plenamente viriles. En el mismo sentido, Guasch (1995) afirma que en el deseo por los jóvenes se valora tanto su masculinidad (la fuerza y la belleza del cuerpo joven), como la indefinición que se deriva de su estado de tránsito hacia lo viril, situación que lo asocia simbólicamente a lo femenino y, por lo tanto, a lo penetrable, lo que se convierte en una confirmación que al interior del sexo entre varones se reproduce una cultura coitocéntrica, pues se asume una réplica de las coordenadas de la heterosexualidad que demandan la penetración como acto central. Sin embargo, estas hipótesis deben validarse con otros trabajos que profundicen los significados que tiene para los varones la iniciación sexual homoerótica en diferentes momentos de su curso de vida. $\curvearrowright$ 


\section{BIBLIOGRAFÍA}

Almaguer, Tomás (1995), "Hombres chicanos: una cartografía de la identidad y del comportamiento homosexual”, en Debate feminista, vol. 11, pp. 46-77.

Amuchástegui, Ana (2000), Virginidad e iniciación sexual en México. Experiencias y significados. México, Edamex, Population Council.

Argüello, Sofia (2013), "Un fantasma ha salido del closet: los procesos de politización de las identidades sexuales en Ecuador y México, 1968-2010". México, El Colegio de México, tesis de doctorado en ciencias sociales con especialidad en sociología.

Bozon, Michel (1998), “Demografía e sexualidade”, en Maria Andréa Loyola (comp.), A sexualidade nas ciencias humanas. Brasil, Universidad del Estado de Rio de Janeiro, pp. 227-251.

Da Silva Brêtas, José, Conceição Vieira da Silva Ohara, Dulcilene Pereira, Wagner de Aguiar y José de Oliveira (2011), “Aspectos da sexualidade na adolescência”, en Ciência y Saúde Coletiva, vol. 16, núm. 7, pp. 3221-3229.

Díez, Jordi (2015), The politics of gay marriage in Latin America: Argentina, Chile y México. New York, Cambridge University Press.

Cáceres, Carlos (1999), La (re)configuración del universo sexual. Cultura(s) sexual(es) y salud sexual entre los jóvenes de Lima a vuelta de milenio. Lima, Universidad Peruana Cayetano Heredia, REDESS Jóvenes.

Figueiredo de Sousa, Lúcia y Romeu Gomes (2009), "Iniciação sexual, masculinidade e saúde: narrativas de homens jovens universitários”, en Ciência y Saúde Coletiva, vol. 14, núm. 2, pp. 653-660.

Fuller, Norma (2001), Masculinidades, cambios y permanencias varones de Cuzco, Iquitos y Lima. Lima, Fondo Editorial PUCP.

Gallego, Gabriel (2010), Demografia de lo otro. Biografias sexuales y trayectorias de emparejamiento 
entre varones en la Ciudad de México. México, El Colegio de México.

Geldstein, Rosa y Marta Schufer (2002), Iniciación sexual y después. Prácticas e ideas de los jóvenes de Buenos Aires. Argentina, Centro de Población CENEP, OMS.

Gubert, Daniela y Valéria Madureira (2009), "Iniciaçao sexual de homens adolescentes”, en Ciência e Saúde Coletiva, vol. 14, núm. 4, pp. 1119-1128.

Heilborn, Maria Luiza y Cristiane Cabral (2006), “As trajetórias homo-bissexuais”, en Maria Luiza Heilborn, Estela Aquino, Michel Bozon y Daniela Knauth (comp.), O aprendizado da sexualidade: reprodução e trajetórias sociais de jovens brasileiros. Rio de Janeiro, Universitária, pp. 361-397.

Juárez, Lucero (2009), "Apropiación de derechos sexuales y reproductivos en la adolescencia: Dimensiones de la ciudadanía”, en La Ventana, vol. 4, núm. 30, pp. 148-180.

Hill, Patricia (1998), "It's all in the family: Intersection of gender, race and nation", en Hypatia, vol. 13, núm. 3, pp. 62-82.

Instituto Nacional de Medicina Legal y Ciencias Forenses (2014), "Exámenes médico-legales por presunto delito sexual", en Forensis 2014 Datos para la vida. Colombia.

Ministerio de Salud y UNFPA (2011), Comportamiento Sexual y Prevalencia del VIH en hombres que tienen relaciones sexuales con hombres en siete ciudades de Colombia. Bogotá.

Necchi, Silvia y Marta Schufer (1999), “Adolescente varón: Iniciación sexual y anticoncepción”, en Revista Chilena de Pediatría, vol. 72, núm. 2, pp. 159-168

Núñez Noriega, Guillermo (2000), Sexo entre varones: Poder y resistencia en el campo sexual. México, Porrúa-PUEG-UNAM.

Rojas, Olga y José Luis Castrejón (2007), "Relaciones de género e iniciación sexual masculina en México”, en Otras miradas, vol. 7, núm. 1, pp. 7-28.

Sandfort, Theo (1998), "Homosexual and bisexual behaviour in european countries”, en Michel Hubert, 
Natalie Bajos y Theo Sandfort (orgs.), Sexual behaviour and HIV/AIDS in Europe. Comparison of National Surveys. Londres, UCL Press, pp. 68-105.

Stern, Claudio, Fuentes-Zurita, Cristina, Lozano-Treviño, Laura Ruth y Fenneke Reysoo (2003), "Masculinidad y salud sexual y reproductiva: un estudio de caso con adolescentes de la Ciudad de México”, en Salud pública de México, vol. 45, suppl.1, pp. 34-43.

Szasz, Ivonne y Susana Lerner (Comps.) (1998), Sexualidades en México. Algunas aproximaciones desde la perspectiva de las ciencias sociales. México, El Colegio de México.

Trujillo, Elvia y Fernando Barrera (2002), “Adolescencia, relaciones románticas y actividad sexual: una revisión”, en Revista Colombiana de Psicología, núm. 11, pp. 115-134.

USAID - México (2007), Estudio TRaC de VIH-SIDA. Evaluación del uso del condón en hombres que tienen sexo con hombres en cinco ciudades mexicanas. Population Services International, Washington, D.C. Disponible en http://psi.hfwebdev.com/resources/research-metrics/publications/hiv/ m\%C3\%A9xico-2007-estudio-trac-de-vihsida-evaluaci\%C3\%B3n-del-uso-d

Vilela, Ana Luiza y Néia Schor (2007), "Homens adolescentes e vida sexual: heterogeneidades nas motivações que cercam a iniciação sexual", en Cadernos de Saúde Pública, vol. 23, núm. 1, pp. 225-234.

\section{Fecha de recepción: 15 de octubre de 2015}

Fecha de aceptación: 25 de enero de 2016

\section{ACERCA DE LOS AUTORES}

\section{GABRIEL GALLEGO MONTES}

(gabriel.gallego@ucaldas.edu.co)

Es doctor en estudios de población por El Colegio de México y profesor-investigador del departamento de Estudios de Familia de la Universidad de Caldas, Manizales, Colombia. Fue galardonado, en 2008, con el premio Gustavo Cabrera que otorga El Colegio de México a la mejor investigación en el campo de la demografía y los estudios de población. Es autor de Demografía de lo otro (El Colegio de México, 
2010), así como de "Implicaciones del VIH-SIDA en la biografía de varones con prácticas homoeróticas en la Ciudad de México", en Salud Pública de México (2010) y de "trayectorias de emparejamiento entre varones en la ciudad de México", en Sexualidades (2010, International Resource Network), entre otros artículos.

\section{SEBASTIÁN GIRALDO AGUIRRE}

(s.giraldoaguirre@gmail.com)

Es maestro en estudios de género por El Colegio de México y sociólogo de la Universidad de Caldas, Manizales, Colombia. Actualmente es docente del departamento de Desarrollo Humano de la Universidad de Caldas. Entre sus áreas de interés destacan las masculinidades, paternidades y sexualidades. Es autor de "Prácticas de paternidad de algunos varones gais de Ciudad de México. Entre tabúes y nuevas apuestas para su ejercicio", en Revista Sociedad y Economía (2015, Universidad del Valle, Colombia). 